\title{
Patient Specific Haemodynamic Modeling after Occlusion Treatment in Leg
}

\author{
T. Gamilov ${ }^{1}$, Yu. Ivanov ${ }^{2}$, P. Kopylov ${ }^{3}$, S. Simakov ${ }^{1}$, Yu. Vassilevski ${ }^{1,2}$ \\ ${ }^{1}$ Moscow Institute of Physics and Technology, 141700, Dolgoprudny, 9 Institutskii Lane, Russia \\ 2 Institute of Numerical Mathematics RAS, 119333, Moscow, 8 Gubkina St., Russia \\ ${ }^{3}$ I.M. Sechenov First Moscow State Medical University, 2-4 Bolshaya Pirogovskaya st. \\ 119991 Moscow, Russia
}

\begin{abstract}
In this work we propose a method for analysis of postsurgical haemodynamics after femoral artery treatment of occlusive vascular disease. Patient specific reconstruction algorithm of $1 \mathrm{D}$ core network based on MRI data is proposed as a tool for such analysis. Along with presurgical ultrasound data fitting it provides effective personalizing predictive method that is validated with clinical observations.
\end{abstract}

Keywords and phrases: haemodynamics, femoral artery occlusion, mathematical modeling, patient specific, 1D core network reconstruction algorithm, presurgical and postsurgical modeling

Mathematics Subject Classification: 35Q92, 76Z05, 65M06

\section{Introduction}

Revascularization is the conventional method of surgical treatment which increases lifetime and life quality for many patients. Balloon angioplasty and stenting are the most advanced techniques of revascularization nowadays. So far no method has been developed for presurgical assessment of revascularization impact to the postsurgical blood flow. Such method would provide a basis for patient specific surgery tactics development. In this work we propose a method of presurgical patient specific analysis of the haemodynamic changes in lower extremities due to atherosclerotic occlusion treatment. It is based on personalized 1D core network reconstruction algorithm, presurgical ultrasound patient specific data fitting and the 1D blood flow simulations.

The basics of our approach to 1D haemodynamic simulation are presented in $[15,17]$. For other works in this field we refer to $[1,5,9,10]$. In this work we address the boundary conditions for the local part of the network. The problem is that haemodynamic parameters in the downstream outlets can not be correctly evaluated presurgically. To cope with this, we impose remote downstream conditions in veins which are disturbed slightly by surgical treatment.

The 1D blood flow simulations require 1D core network representation of the 3D patient specific vascular network. This motivates importance of an algorithm which produces the set of $1 \mathrm{D}$ edges (cores)

*Corresponding author. E-mail: simakov@crec.mipt.ru 
and junction nodes on the basis of 3D vascular domain extracted from MRI data. A few software tools provide partly such functionality. The most popular tools are Amira ${ }^{\circledR}$ [20] and VMTK [19]. In this work we use VMTK for 3D volume extraction from MRI data for meshing and reconstruction vessel centerlines. The final 1D core network is produced by a new algorithm capable to process general networks with loops.

The outline of the paper is as follows. We briefly introduce the mathematical model in section 2.1. Patient specific network reconstruction tools are presented in section 2.2. Additional patient specific fitting issues are particularly addressed in section 2.3. The results of patient specific haemodynamic modeling after occlusion treatment in leg are presented in section 3. The discussion of the presented approach and the future work constitutes section 4 .

\section{Methods}

\subsection{D network model for haemodynamics}

\subsubsection{Core model}

As a core model for the closed blood circulation we used 1D network dynamical model [15-17] accounting for arterial and venous parts of the thigh sub-network. The model considers the flow of viscous incompressible fluid in the network of elastic tubes/vessels. The flow in every vessel is described by mass and momentum balance equations

$$
\begin{array}{r}
\partial S / \partial t+\partial(S u) / \partial x=0 \\
\partial u / \partial t+\partial\left(u^{2} / 2+p / \rho\right) / \partial x=f_{t r}(S, u),
\end{array}
$$

where $t$ is the time; $x$ is the coordinate along the vessel; $\rho$ is the blood density (constant); $S(t, x)$ is the vessel cross-section area; $S^{0}$ is the unstressed cross-section area; $u(t, x)$ is the linear velocity of blood flow averaged over the cross-section; $p$ is the blood pressure; $f_{t r}$ is the friction force given in the $k$-th vessel by

$$
f_{t r}\left(S_{k}, u_{k}\right)=-\frac{4 \pi \mu u_{k}}{S_{k} \tilde{S}_{k}}\left(\tilde{S}_{k}+\tilde{S}_{k}^{-1}\right)
$$

$\mu$ is the coefficient of friction, $\tilde{S}_{k}=S_{k} / S_{k}^{0}$.

At the vessels junctions the Poiseuille pressure drop and the mass conservation conditions [15] are applied (for motivation we refer to section 4):

$$
\begin{gathered}
p_{k}\left(S_{k}\left(t, \tilde{x}_{k}\right)\right)-p_{\text {node }}^{l}(t)=\varepsilon_{k} R_{k}^{l} S_{k}\left(t, \tilde{x}_{k}\right) u_{k}\left(t, \tilde{x}_{k}\right), k=k_{1}, k_{2}, \ldots, k_{M}, \\
\sum_{k=k_{1}, k_{2}, \ldots, k_{M}} \varepsilon_{k} S_{k}\left(t, \tilde{x}_{k}\right) u_{k}\left(t, \tilde{x}_{k}\right)=0,
\end{gathered}
$$

where $M$ is the number of vessels meeting at node with index $l ;\left\{k_{1}, \ldots, k_{M}\right\}$ is the range of the indexes of these vessels; $p_{\text {node }}(t)$ is the pressure at the junction node; $\varepsilon=1, \tilde{x}_{k}=L_{k}$ for incoming vessels, $\varepsilon=-1, \tilde{x}_{k}=0$ for outgoing vessels; $R_{k}^{l}$ is the hydraulic resistance. The system of equations $(2.4)-(2.5)$ is closed by finite difference approximation of compatibility conditions along outgoing characteristics. The order of the respective junction system can be reduced from $2 M+1$ to $M$ equations and effectively solved by Newton method $[15,17]$.

The elastic properties of the vessel wall are given by the state equation:

$$
p\left(S_{k}\right)-p_{* k}=\rho c_{k}^{2} f\left(S_{k}\right),
$$

where S-like function $f\left(S_{k}\right)$ is approximated as

$$
f\left(S_{k}\right)= \begin{cases}\exp \left(\tilde{S}_{k}-1\right)-1, & \tilde{S}_{k}>1 \\ \ln \tilde{S}_{k}, & \tilde{S}_{k} \leqslant 1\end{cases}
$$


$p_{* k}$ is the pressure in the tissues surrounding the vessel, $c_{k}$ is the velocity of small disturbance propagation in the vessel wall which also has meaning of pulse wave velocity (PWV) in the unstressed vessel [18] characterizing material stiffness.

Details on numerical implementation of this model are discussed in $[14,15,17]$.

\subsubsection{Boundary conditions}

Since we consider a local region of the thigh vasculature, we should address the boundary conditions at the vascular network inlet and outlet.

The entry point of the simulated vessels network corresponds to the beginning of common iliac artery (see Figure 1 and vessel 1 in Figure 9). Patient specific data for this node were unavailable for us and we set the inlet boundary conditions as

$$
u(t, 0) S(t, 0)=\alpha Q_{H}(t),
$$

where $Q_{H}(t)$ is heart ejection profile simulated by four-chamber dynamical heart model [15]. We approximate common iliac artery blood flow inlet profile by scaling heart ejection profile with coefficient $\alpha$. The lower estimate of $\alpha$ for the common iliac artery is based on the estimates for blood flow through the femoral artery $635 \mathrm{ml} / \mathrm{min}$ [6], heart rate 60 strokes/min and stroke volume $60 \mathrm{ml}$ :

$$
\alpha>\frac{635 \mathrm{ml} / \mathrm{min}}{60 \mathrm{str} / \mathrm{min} \cdot 60 \mathrm{ml} / \mathrm{str}} \approx \frac{1}{6} .
$$

The lower estimate is in a good agreement with the value computed in the model of global systemic circulation [15]. In this work we take $\alpha=0.21$ as it provides the best fit between calculated and measured presurgical velocity maximum in the femoral artery (see Table 2). The same inlet boundary condition is applied for postsurgical state simulations as it is distanced upstream from the surgical region.

The outlet boundary conditions at the terminal points of the vessels $6,8,9,11,13,14$ (Figure 9) can be based on presurgically measured velocities in the particular points given in Table 2. However, our objective is to predict haemodynamic changes presurgically and we can not rely on postsurgical measurements. Moreover, the outlet boundaries are placed downstream and blood flow may be changed significantly due to surgical intervention. Therefore, we have to introduce conditions which are slightly disturbed by postsurgical blood flow redistribution. We connect the averaged venous network of the same structure (see Figure 9) to the terminal outlet points of the considered arterial region. Vein diameters are set two times greater and coefficients $c_{k}$ are set two times less than the respective values for the arteries. This results in accumulation of four times more blood in venous part and makes the venous wall more soft and flexible. Since we neglect pulsations in veins, we set at the terminal point of the venous network a steady blood flow as the boundary condition. In order to maintain the mass balance in the total network, we make this flow equal to the time-averaged blood flow at the entry point of the arterial part

$$
u(t, L) S(t, L)=\alpha \int_{t}^{t+T_{H}} Q_{H}(t) d t,
$$

where $Q_{H}(t)$ is taken from $(2.8), T_{H}$ is the period of the cardiac cycle.

\subsection{Reconstruction of patient specific $1 \mathrm{D}$ core network}

1D blood flow network simulations require 1D core network reconstruction. The overall algorithm of reconstruction is divided into the following steps: 1) 3D volume segmentation of vascular structure, 2) meshing and centerlines extraction, 3) centerlines merging, and 4) network reconstruction.

We should note that commercial software Amira ${ }^{\circledR}$ provides similar functionality. It helps to perform semi-automatic blood vessels segmentation, to produce skeletonisation based on distance map and thinning methods for a connected set of voxels, and to generate centerlines on the basis of calculation of Euclidean distance to the nearest boundary for every point in the vascular 3D domain. 
In this work we propose a method which is based on the open source library VMTK. This library can be modified and can be easily extended with new methods. We use VMTK to produce centerlines of 3D vascular domain extracted from MRI data and extend VMTK with the new algorithm for graph reconstruction. To perform vascular domain extraction, we filter input data and eliminate bones, air and surrounding tissues from the original image by thresholding. Level set method is used for tracking vascular branches and marching cubes method is used to extract the surface of vessels. The result of this preprocessing of patient specific MRI data is presented in Figure 1.

$1 \mathrm{D}$ core network is produced on the basis of centerlines which represent the $3 \mathrm{D}$ vascular domain. Several methods of centerlines generation are reviewed and compared in $[4,8]$. We use the method implemented in VMTK which is based on Voronoi diagrams [2]. The results of meshing by marching cubes and centerlines computation are presented in Figure 2.

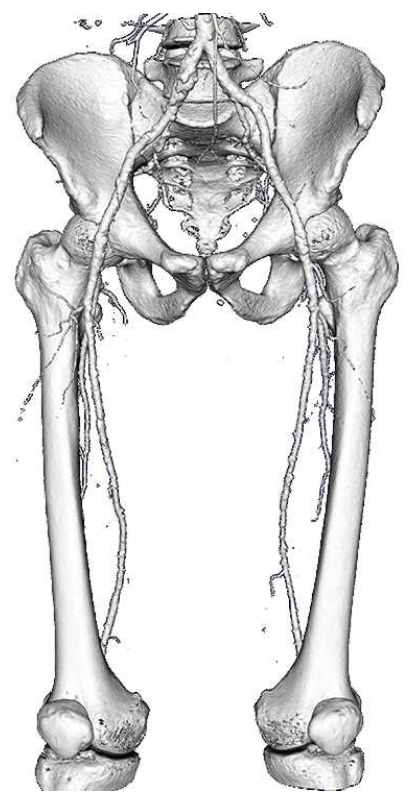

A

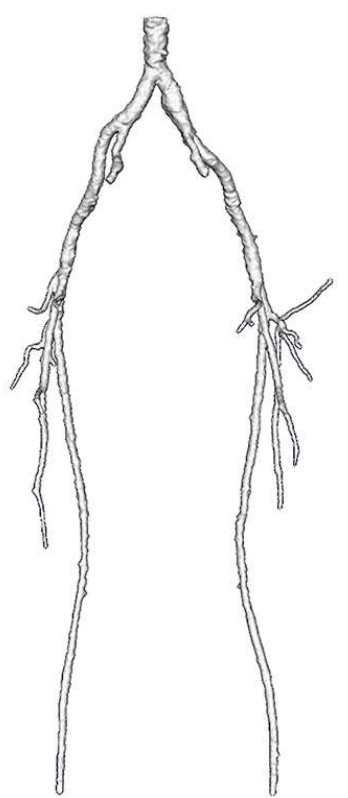

$\mathrm{B}$

Figure 1. 3D segmentation based on MRI data: A — vessels with bones, B — vessels.

The resulting 1D core network is described by a set of nodes and a set of edges (cores) connecting nodes. These data can be produced from centerline representation of the initial 3D vascular domain. Every centerline is described by an ordered set of pairs $\left\{\left(\mathbf{a}_{i}, r_{i}\right)\right\}_{i=1}^{n}$ where $\mathbf{a}_{i}=\left(x_{i}, y_{i}, z_{i}\right)$ is the radius vector of the central point in the vessel cross-section and $r_{i}$ is the mean radius of the vessel at this point. These centerlines should be merged and segmented with junction points.

To present algorithms of $1 \mathrm{D}$ core network reconstruction, we take advantage of the similarity between the vascular network and a tree-like structure with branches, junctions and root. However, our core networks may have closed loops and graph terminology seems to be more appropriate for our algorithmic description. Further in this section we shall deal with sets of graph nodes and sets of graph branches. We ascribe to every graph node a point $A_{i}$ in the $3 \mathrm{D}$ space with corresponding radius vector $\mathbf{a}_{\mathbf{i}}=\left(x_{i}, y_{i}, z_{i}\right)$. The branch object contains a pair of incident nodes, the length of the corresponding vessel segment (along the centerline) and averaged radius.

Every centerline computed with VMTK goes from every chosen inlet to every outlet. For further purposes we should split the centerlines by eliminating coincided parts. In Figure 3 we show a set of 

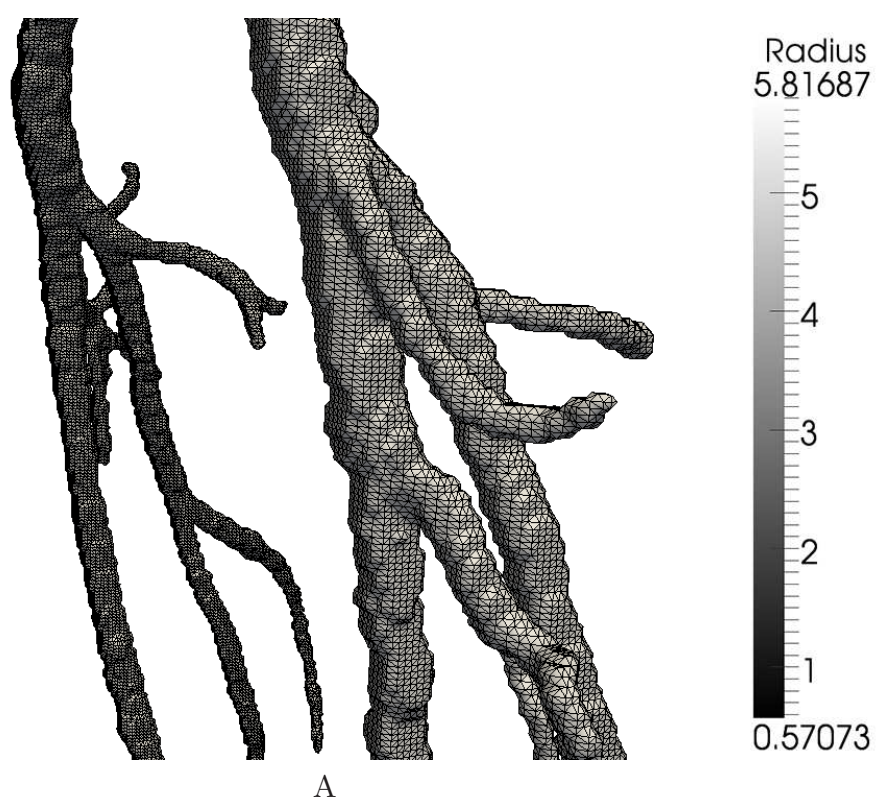

Figure 2. 3D vascular domain: A - polygonal mesh, B - computed centerlines.

centerlines corresponding to branch ends $[O, A],[O, B],[O, C],[O, D]$ before splitting and new split and merged set $[O, A],\left[O_{1}, B\right],\left[O_{2}, C\right],\left[O_{3}, D\right]$.

Centerlines intersection is determined as follows. Centerline $C^{\prime}=\left\{\left(\mathbf{a}_{i}^{\prime}, r_{i}^{\prime}\right)\right\}_{i=1}^{n^{\prime}}$ intersects centerline $C=\left\{\left(\mathbf{a}_{i}, r_{i}\right)\right\}_{i=1}^{n}$ if

$$
\exists \mathbf{a}^{\prime} \in\left\{\mathbf{a}_{1}^{\prime}, \mathbf{a}_{n}^{\prime}\right\}, \exists k \in\{1, . ., n-1\}:\left|\mathbf{a}^{\prime}-\mathbf{a}^{*}\right| \leq r_{k+0.5}\left(\mathbf{a}^{*}\right)
$$

where $\mathbf{a}^{*}$ is the projection of $\mathbf{a}^{\prime}$ onto $\left[\mathbf{a}_{k}, \mathbf{a}_{k+1}\right]$ and $r_{k+0.5}$ is linearly interpolated radius

$$
\begin{gathered}
r_{k+0.5}\left(\mathbf{a}^{*}\right)=\left\{\begin{array}{r}
r_{k}\left(1-\lambda_{k+0.5}\left(\mathbf{a}^{*}\right)\right)+r_{k+1} \lambda_{k+0.5}\left(\mathbf{a}^{*}\right), \mathbf{a}^{*} \in\left[\mathbf{a}_{k}, \mathbf{a}_{k+1}\right] \\
0, \mathbf{a}^{*} \notin\left[\mathbf{a}_{k}, \mathbf{a}_{k+1}\right],
\end{array}\right. \\
\lambda_{k+0.5}\left(\mathbf{a}^{*}\right)=\frac{\left|\mathbf{a}^{*}-\mathbf{a}_{k}\right|}{\left|\mathbf{a}_{k+1}-\mathbf{a}_{k}\right|} .
\end{gathered}
$$

We shall refer to $\mathbf{a}^{*}$ as the branching point (Figure 4). 


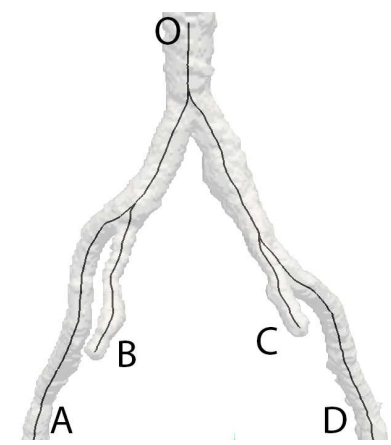

a)

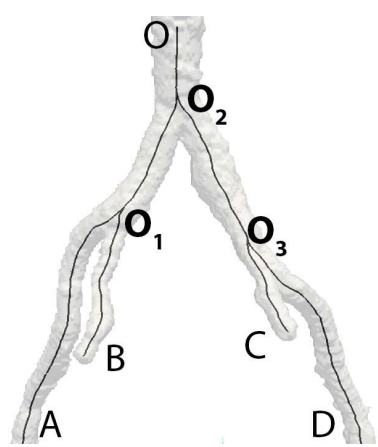

b)

Figure 3. Centerlines before (a) and after (b) splitting.

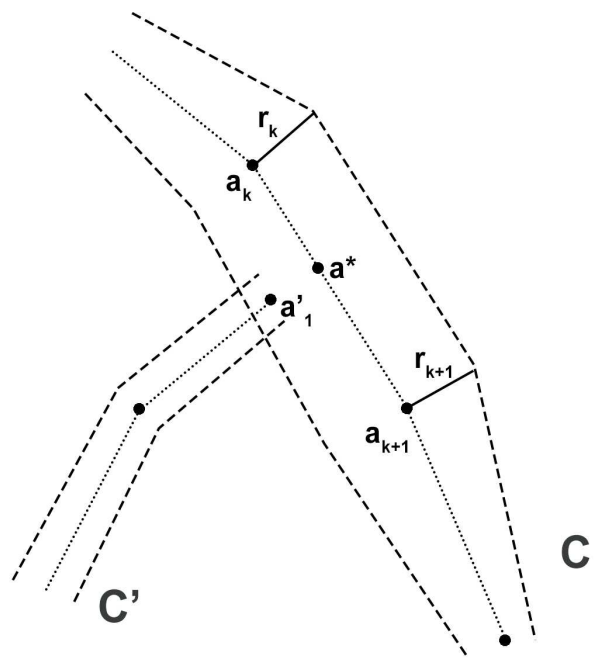

FIGURE 4. Intersection of centerlines.

We present the first algorithm assuming that the graph to be reconstructed has no loops, i.e. possesses a tree-like structure. The algorithm is initialized by choosing the root centerline $C_{1}=\left\{\left(\mathbf{a}_{i}, r_{i}\right)\right\}_{i=1}^{n}$. The other centerlines are checked for the intersection with the root centerline and branching points are determined for every intersection. Then the algorithm recursively proceeds to examine the centerlines intersecting the root considering them subsequently as new roots.

Let $\mathcal{M}=\left\{C_{1}, C_{2}, . ., C_{m}\right\}$ be the set of input centerlines, $\mathcal{N}, \mathcal{B}$ be the output node and branch arrays, respectively, $\mathcal{N}$ be the array of radius vectors of branching points and $\mathcal{B}$ be the array of incident node indexes, $\# \mathcal{N}$ denote the cardinality of set $\mathcal{N}$. Let $\mathcal{R}$ and $\mathcal{P}$ be work arrays, $\mathcal{R}$ be a set of pairs $(C, k)$ where $C$ is the current root centerline and $k$ is a graph vertex index corresponding to the branching point. Let $\mathcal{P}$ be a temporal array of the current root centerline and the radius vector of the branching point. The algorithm starts with choosing the initial (root) centerline $C_{1}$ and its first node $\mathbf{a}_{1}$ to be the root node with index 1 . The main loop of the algorithm picks the centerlines from set $\mathcal{R}$. 


\section{Algorithm 2.1.}

1: Set $\mathcal{R}=\left\{\left(C_{1}, 1\right)\right\}, \mathcal{N}=\left\{\mathbf{a}_{1}\right\}, \mathcal{B}=\{\}$.

2: while $\mathcal{R} \neq \varnothing$ do

Extract pair $(R, I)$ from $\mathcal{R}$. Find all centerlines intersecting $R$ :

for $M \in \mathcal{M} \backslash R$ do

$\mathbf{a}^{*}=M \cap R$

if $\mathrm{a}^{*} \neq \varnothing$ then end if

Add pair of intersecting centerline and branching point $\left(M, \mathbf{a}^{*}\right)$ to $\mathcal{P}$

end for

Order $\mathcal{P}$ according to distances from branching points to point $\mathcal{N}[I]$

Set $n_{1}=I$. Add the detected branches and graph nodes

for $j=1 \rightarrow \# \mathcal{P}$ do

$(M, \mathbf{a})=\mathcal{P}[j]$

Append a to $\mathcal{N}$

Set $n_{2}=\# \mathcal{N}$

Append $\left(n_{1}, n_{2}\right)$ to $\mathcal{B}$

Set $n_{1}=\# \mathcal{N}$

Add $\left(M, n_{1}\right)$ to the root array $\mathcal{R}$

end for

Append $\mathbf{a}_{n}^{r}$, the endpoint of $R$, to $\mathcal{N}$

Set $n_{2}=\# \mathcal{N}$

Append $\left(n_{1}, n_{2}\right)$ to $\mathcal{B}$

Remove $R$ from $\mathcal{M}$

end while

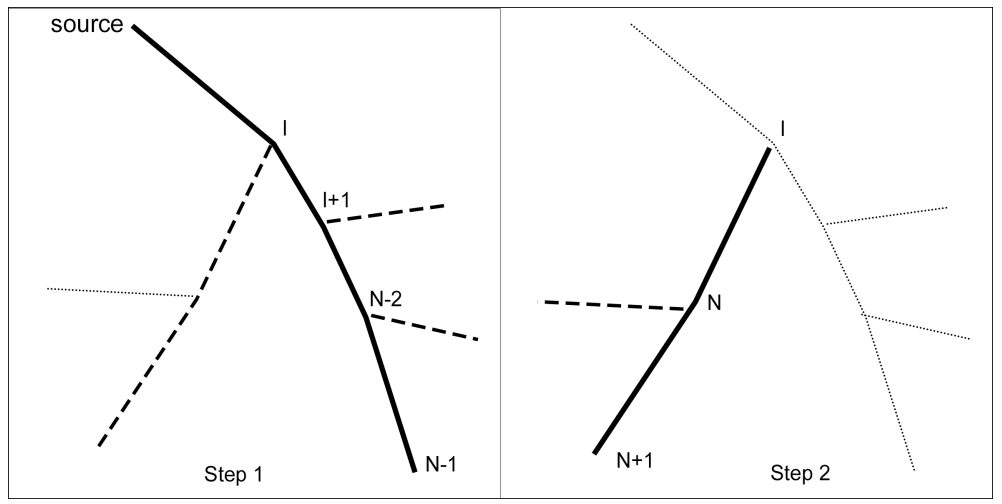

Figure 5. Nodes numbering in algorithm 2.1.

This algorithm describes hierarchical traversing (Figure 5) through $\mathcal{M}$ with elimination of visited elements from set $\mathcal{M}$ (algorithm 2.1, line 23). The assumption of tree-like topology implies that on each iteration of the main loop it holds

$$
\forall M \in \mathcal{M} \backslash R: R \cap M=\varnothing .
$$

In general case this condition can be violated. In this case it is necessary to remain visited elements in the list of centerlines. We can generalize algorithm 2.1 for arbitrary graph with loops using one more work array $\mathcal{W}$ and adding the lines 14-17, 29 


\section{Algorithm 2.2.}

1: Set $\mathcal{R}=\left\{\left(C_{1}, 1\right)\right\}, \mathcal{N}=\left\{\mathbf{a}_{1}\right\}, \mathcal{B}=\{\}, \mathcal{W}=\{\}$

2: while $\mathcal{R} \neq \varnothing$ do

3:

Extract pair $(R, I)$ from $\mathcal{R}$

for $M \in \mathcal{M} \backslash R$ do

$\mathbf{a}^{*}=M \cap R$

if $\mathrm{a}^{*} \neq \varnothing$ then

end if

$\operatorname{Add}\left(M, \mathbf{a}^{*}\right)$ to $\mathcal{P}$

end for

Order $\mathcal{P}$ according to distances from branching points to point $\mathcal{N}[I]$

Set $n_{1}=I$

for $j=1 \rightarrow \# \mathcal{P}$ do

$$
\begin{aligned}
& (M, \mathbf{a})=\mathcal{P}[j] \\
& \text { if } M \in\left\{C \mid\left(C, I_{c}\right) \in \mathcal{W}\right\} \text { then }
\end{aligned}
$$

$\left(M, I_{m}\right) \in \mathcal{W}$

Append $\left(n_{1}, I_{m}\right)$ to $\mathcal{B}$

Set $n_{1}=I_{m}$

else

Append a to $\mathcal{N}$

Set $n_{2}=\# \mathcal{N}$

Append $\left(n_{1}, n_{2}\right)$ to $\mathcal{B}$

Set $n_{1}=\mathcal{N}$

end if

$\operatorname{Add}\left(M, n_{1}\right)$ to $\mathcal{R}$

end for

Append $\mathbf{a}_{n}^{r}$, the endpoint of $R$, to $\mathcal{N}$

Set $n_{2}=\# \mathcal{N}$

Append $\left(n_{1}, n_{2}\right)$ to $\mathcal{B}$

Add $\left(R, n_{2}\right)$ to $\mathcal{W}$

\section{end while}

Note that for each iteration we leave the current centerline $R$ in $\mathcal{M}$ but do not add the element to $\mathcal{R}$ if it was visited earlier. For this purpose we use work array $\mathcal{W}$. Item of $\mathcal{W}$ consists of visited element and graph node index corresponding to the endpoint. Thus when visited element intersects the current root, a new node is not added (Figure 6).

Both algorithms produce graph with node degree not greater than 3 . The reason is that the algorithms can generate branches with too small length. To cope with this, we define the minimal branch length value $\Delta$. We choose $\Delta(\mathbf{x}) \in[2 r(\mathbf{x}), 4 r(\mathbf{x})]$, where $r(\mathbf{x})$ is local vessel radius, i.e. the radius corresponding to the centerline point $\mathbf{x}$. We consider the set of branching points $\left\{\mathbf{a}_{i}\right\}$. If

$$
\exists \mathbf{a}_{i}, . ., \mathbf{a}_{i+k}: \sum_{j=0}^{k-1}\left|\mathbf{a}_{i+j+1}-\mathbf{a}_{i+j}\right|<\Delta,
$$

and

$$
\nexists \mathbf{a}_{i-1}, \mathbf{a}_{i+k+1}:\left|\mathbf{a}_{i}-\mathbf{a}_{i-1}\right|<\Delta, \quad\left|\mathbf{a}_{i+k+1}-\mathbf{a}_{i+k}\right|<\Delta,
$$

then we replace $\mathbf{a}_{i}, . ., \mathbf{a}_{i+k}$ with the node

$$
\mathbf{a}_{i}=\ldots=\mathbf{a}_{i+k}=\frac{1}{k} \sum_{j=0}^{k-1} \mathbf{a}_{i+j} .
$$




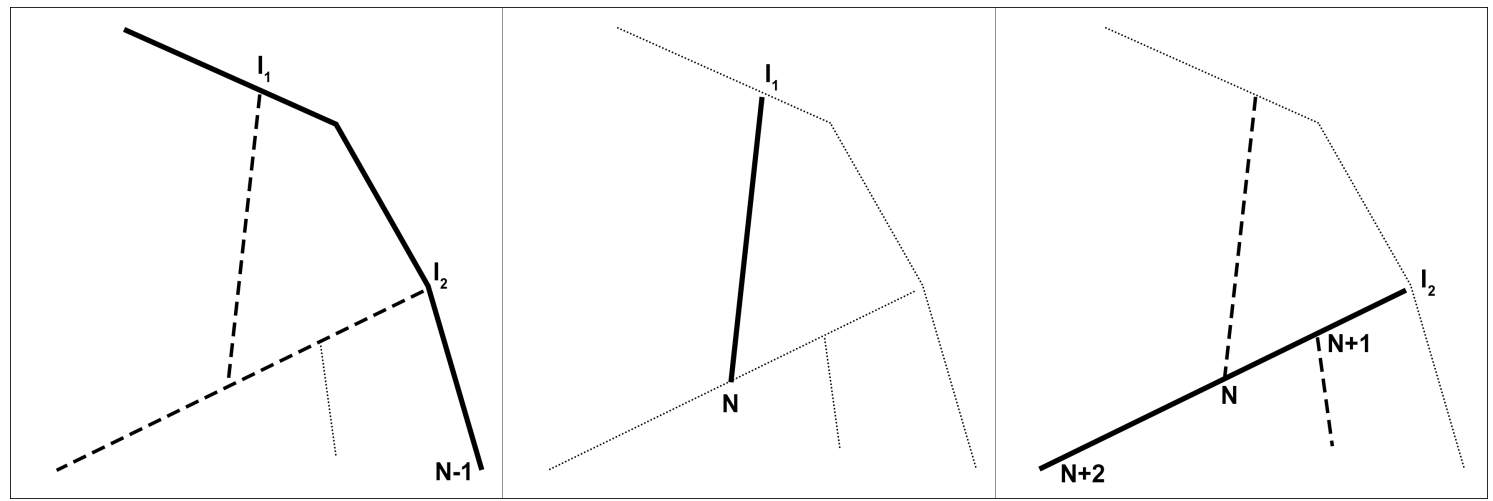

FiguRE 6. Nodes numbering in algorithm 2.2.

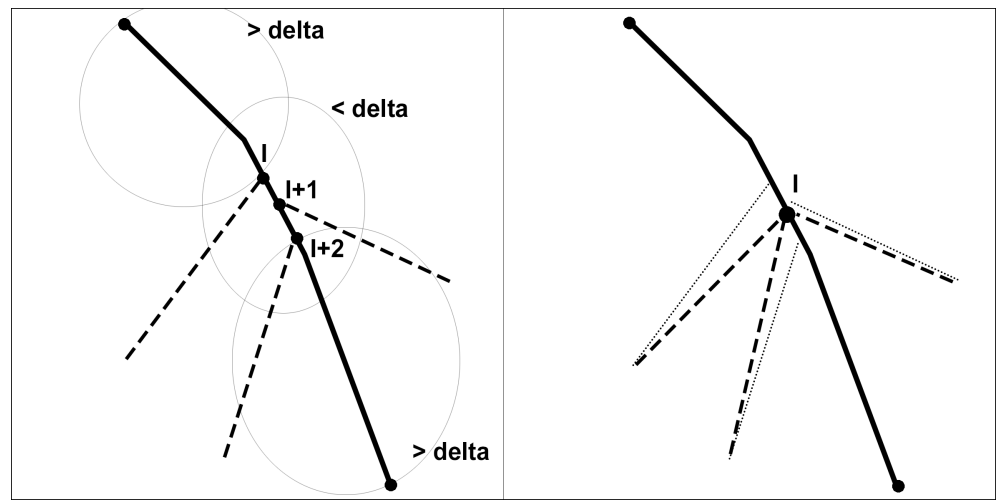

FIgURE 7 . Merging of nodes due to minimal branch length threshold.

The processes is illustrated in Figure 7. In this case only one node is added to the set of graph nodes.

The complexity of algorithm 2.2 is $O\left(M^{2} N\right)$ where $M$ is the number of centerlines, $N$ is the maximal number of points in centerline. The computational complexity of algorithm 2.1 is $O(M N K)$ where $K$ is the number of branching levels. Evidently $K \leqslant M$. The computational cost can be reduced by filtering off points along the centerline which fail to satisfy

$$
\forall \mathbf{a}_{i}, \mathbf{a}_{i+1} \in C=\left\{\left(\mathbf{a}_{i}, r_{i}\right)\right\}_{i=1}^{n} \Rightarrow\left|\mathbf{a}_{i+1}-\mathbf{a}_{i}\right|>\Delta_{i+0.5} .
$$

Algorithm 2.1 was applied to the MRI data of arteries in the particular region shown in Figure 1. The result is presented in Figure 9. No appropriate MRI data are available for the total vascular network. Therefore we tested algorithm 2.2 for the total closed vessel network [21], a model 3D vascular network designed as an educational tool. The resulting 1D core network for systemic arteries is presented in Figure 8.

\subsection{Patient specific model fitting}

We apply the above approach to 1D core network reconstruction to predict changes of local haemodynamics in thigh vasculature due to the occlusion treatment in the femoral artery. Processing of patient specific MRI data results in 1D core arterial network shown in Figure 9. Geometrical parameters of the network (vessels lengths and diameters) are defined by our 1D core network reconstruction algorithm and correspond to the patient specific morphology. 

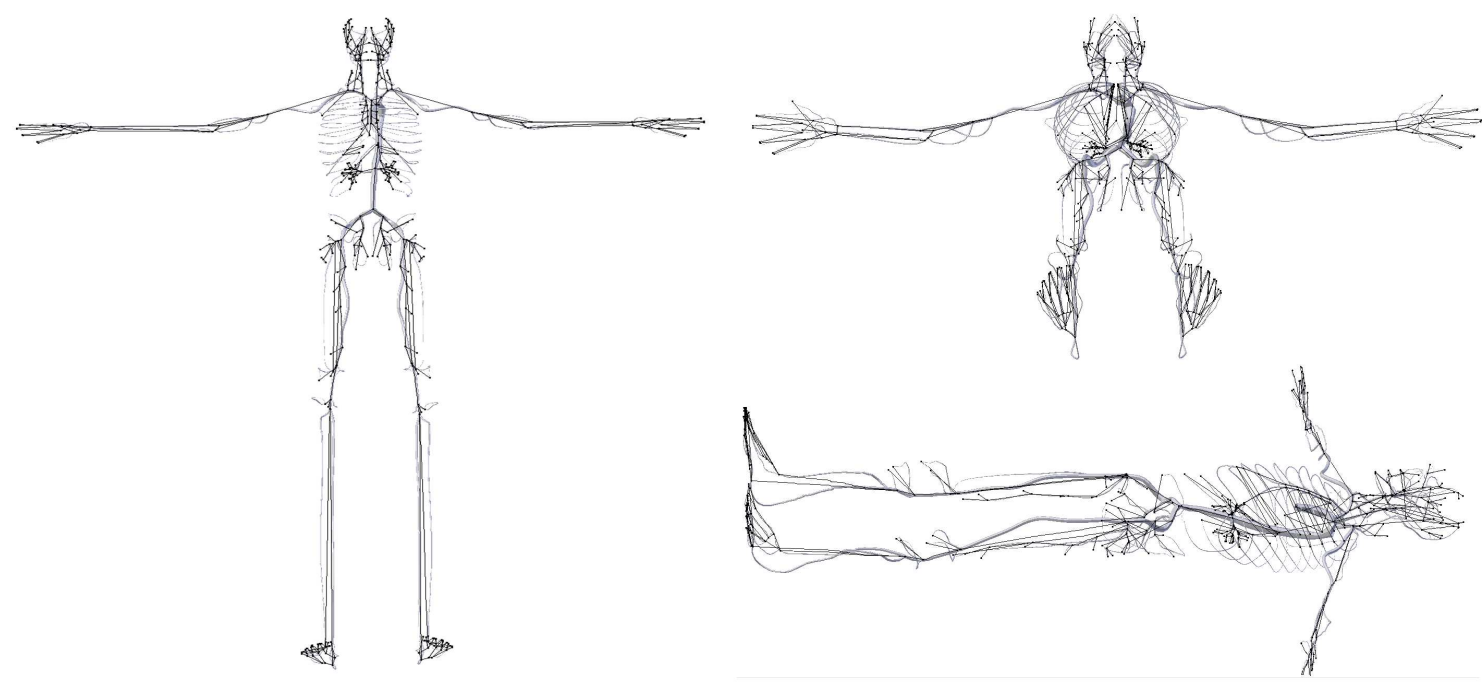

FiguRE 8 . The 1D core network of arterial part of systemic circulation based on virtual $3 \mathrm{D}$ model [21].

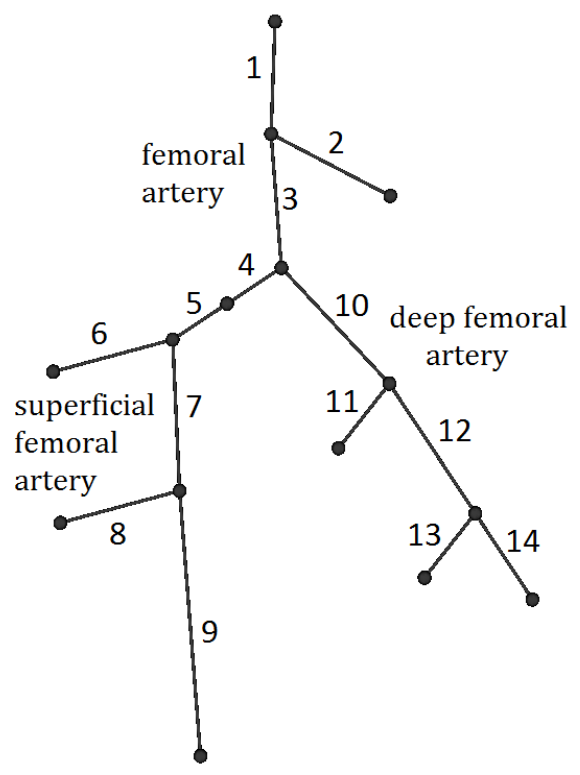

FiguRE 9. The large arterial vessels network of the left thigh.

Functional parameters $c_{k}$ in (2.6) and $R_{k}$ in (2.4) are unavailable in the case of study. We assess their acceptable ranges basing on general physiological and anatomical data [7,11-13], as well as patient parameters such as height, weight, medical history and the following assumptions:

1. Vessels 1, 3, 4, 5, 7 and 9 (Figure 9) are the parts of the femoral artery and its descendant popliteal artery. These main leg arteries have relatively high stiffness $c_{k}$ and low resistance $R_{k}$ since they carry large bulk of blood. 


\begin{tabular}{|c|c|c|c|c|}
\hline$k$ & $l_{k}, \mathrm{~cm}$ & $d_{k}, \mathrm{~cm}$ & $c_{k}, \mathrm{~cm} / \mathrm{s}$ & $R_{k}, \mathrm{ba} \cdot \mathrm{s} / \mathrm{cm}^{3}$ \\
\hline 1 & 4.63 & 1.25 & 1200 & 100 \\
2 & 6.51 & 0.72 & 700 & 680 \\
3 & 14.09 & 0.94 & 1200 & 100 \\
4 & 1.0 & 0.93 & 840 & 70 \\
$5^{*}$ & 0.79 & 0.93 & 840 & 70 \\
$5^{* *}$ & 0.79 & 0.37 & 840 & 210 \\
6 & 4.11 & 0.45 & 700 & 1400 \\
7 & 2.08 & 0.84 & 980 & 10 \\
8 & 8.07 & 0.41 & 700 & 10 \\
9 & 38.75 & 0.61 & 980 & 60 \\
10 & 1.67 & 0.83 & 700 & 120 \\
11 & 4.50 & 0.46 & 700 & 56 \\
12 & 8.17 & 0.63 & 700 & 72 \\
13 & 3.55 & 0.45 & 700 & 80 \\
14 & 12.70 & 0.44 & 700 & 80 \\
\hline
\end{tabular}

TABle 1. Parameters of the arterial part of the network: $l_{k}$ is the length of the $k^{\text {th }}$ vessel, $d_{k}$ is the diameter of the $k^{\text {th }}$ vessel, ${ }^{*}$ denotes the vessel without occlusion, ${ }^{* *}$ denotes the vessel with occlusion.

2. Vessels 2 and 6 (Figure 9) start from the greater iliac and the femoral arteries with substantial angles in junction that results in increased resistances $R_{k}$.

Parameter values are fitted to match the available presurgical Doppler ultrasound measurements at some points before and after occlusion. The parameters are presented in Table 1. We assume these parameters to remain the same after the occlusion treatment since according to numerical evidence the Reynolds number is not changed significantly in the most vessels. The only exception is vessel 5 which is a fragment of the femoral artery where the occlusion is introduced. Parameters of vessel 5 in presurgical simulations are obtained by modifications of parameters of healthy vessel 4: the hydraulic resistance is increased by factor of 3, the diameter and the lumen $S_{0}$ in (2.6) are decreased by $60 \%$ and $84 \%$, respectively. Parameters of vessel 5 in postsurgical simulations are equal to the respective parameters for the healthy vessel 4.

\section{Results}

Two series of numerical experiments have been performed. They simulate haemodynamics in large thigh arteries before and after femoral artery occlusion treatment.

The first set of numerical simulations was used to fit unmeasurable functional parameters $c_{k}$ and $R_{k}$. The result of $c_{k}$ and $R_{k}$ identification is given in Table 1 . We adjusted these parameters basing on the best possible fitting between calculated and measured peak velocities in a set of measurement points. The result of fitting is presented in the column presurgical in Table 2 . We note that even better matching can be achieved by further adjustment of the vessels parameters shifting them to non-physiological ranges. For instance, the model peak velocity in the deep femoral artery (see Table 2) could be increased by increasing $c_{k}$. This would give elasticity value of the deep femoral artery less than elasticity of the femoral artery that violates our assumptions stated in section 2.3.

The second set of numerical simulations considers postsurgical blood flow through the left thigh arteries after occlusion treatment. The same parameters values were used in this case except for vessel 5 , see Table 1. We compare the computed model results and measured values presented in the column postsurgical in Table 2. We observe quite good coincidence between these data sets: the maximum relative error is not greater than $20 \%$. The largest error is observed in the distal part of the superficial femoral 


\begin{tabular}{|c|c|c|c|c|}
\hline \multirow{2}{*}{ Measurement points (see Figure 9) } & \multicolumn{3}{|c|}{ Peak blood velocity $(\mathrm{cm} / \mathrm{s})$} \\
\cline { 2 - 5 } & presurgical & \multicolumn{2}{c|}{ postsurgical } \\
\cline { 2 - 5 } & patient & model & patient & model \\
\hline 3 - common femoral art. & 148 & 149 & 150 & 155 \\
4 - superficial femoral art. (proximal) & 48 & 54 & 65 & 70 \\
12 - deep femoral art. & 103 & 93 & 69 & 83 \\
5 - occlusion & $>300$ & 340 & - & 71 \\
7 - superficial femoral art. (distal) & - & 67 & 98 & 86 \\
9 - popliteal art. & 52 & 56 & 72 & 72 \\
\hline
\end{tabular}

TABLE 2. Peak blood velocities.

artery. The error is ascribed to the physiological limitations during parameters fitting procedure and a possible input data error discussed in section 4.

\section{Discussion}

The proposed method of $1 \mathrm{D}$ core network reconstruction is competitive to the methods implemented in the well-known commercial software [20]. It also has some advantages over the open-source algorithms [19]. In particular, our reconstruction algorithm is capable to handle general graphs without tree-like topology restriction.

Table 2 shows that the model data match well the measurements obtained from the patient. We ascribe the $20 \%$ error observed in the distal part of the superficial femoral artery to insufficient MRI resolution. General anatomy [7] states that one of the deep femoral artery branches has a connection with the popliteal artery which provides an alternate pass in the case of femoral artery occlusion. We failed to observe this connection in patient MRI data. Being introduced to the model, the alternate pass would decrease the peak velocity due to the collateral flow thus decreasing error.

We should note that vessels with atherosclerotic occlusion are characterized by higher stiffness. We increased the stiffness in physiologically reasonable range but did not observe a significant impact on the computational results.

Formally, boundary conditions at the junction nodes should be derived from limiting ratios in (2.1), (2.2) that reduce to the total pressure conservation in pseudo-steady approximation. On the other hand, many vessel junctions have complex flow profiles that can be described accurately only via 3D FSI simulations. Due to high computational complexity the straightforward approach fails to describe more than several junctions. Dealing with 1D approach we use mass conservation condition (2.5) and Poiseuille's pressure drop condition (2.4). This approach was discussed in more detail in [15].

One of the most difficult issues in this and similar works is to fit values of $R_{k}$ and $c_{k}$ or to recover explicitly vessel wall elasticity characteristic $P(S)$. Available medical equipment does not allow to measure these values in regular way. We have to fit them in physiological range to conform with Doppler ultrasound data. Such procedure introduces uncertainties to the results. Therefore, the method should be analyzed and verified on statistically sufficient number of patients.

In this work we focused on the haemodynamic analysis of the atherosclerotic occlusion treatment in the femoral artery. The same method can be applied to the other parts of the vascular system and other angiosurgical procedures such as cava filter implantation and artificial embolisation of arterial-venous malformations that will be parts of our future work.

Acknowledgements. We would like to acknowledge the staff of I.M.Sechenov First Moscow State Medical University and especially A.B. Tagiltsev.

This work was partially supported by the RFBR grants 12-01-33084, 14-01-00779, 14-01-00830, 14-01-00036,

Russian Federation President Grant for Government Support of Young Russian Scientists MK 2719.2012.9.

Section 2.1.1. was only supported by the Russian Scientific Foundation Grant No 14-11-00877 


\section{References}

[1] N. V. Abakumov, S. I. Mukhin, A. P. Favorski, et. al. Strategy of mathematical cardiovascular system modeling. Matem. Mod., 12 (2000), 106-117.

[2] L. Antiga. Patient-specific modeling of geometry and blood-flow in large arteries. PhD thesis. Politecnico di Milano, Milan, 2003.

[3] L. Antiga, D. A. Steinman. Robust and objective decomposition and mapping of bifurcating vessels. IEEE Transactions on Medical Imaging, 23 (2004), 704-713.

[4] K. T. Diedrich, J. A. Roberts, R. H. Schmidt, D. L.Parker. Comparing performance of centerline algorithms for quantitative assessment of brain vascular anatomy. Anat Rec (Hoboken), 295 (2012), 2179.

[5] L. Formaggia, A. Quarteroni, A. Veneziani. Cardiovascular mathematics. DE: Springer, Heidelberg, 2009.

[6] V. Ganz, A. Hlavova, A. Fronek, J. Linhart, I. Prerovsky. Measurement of blood flow in the femoral artery in man at rest and during exercise by local thermodilution. Circulation, 30 (1964), 86-89.

[7] S. Standring. Gray's Anatomy: The Anatomical Basis of Clinical Practice, 40th ed., Elsevier, Churchill-Livingstone, 2008.

[8] J. Liu, K. Subramanian. Accurate and robust centerline extraction from tubular structures in medical images. In: Advances in Information and Intelligent Systems, part 2, Vol. 251, Springer Berlin Heidelberg, 2009, 139-162.

[9] L. O. Müller, C. Parés, E. F. Toro. Well-balanced high-order numerical schemes for one-dimensional blood flow in vessels with varying mechanical properties. J. Comp. Phys., 242 (2013), 53-85.

[10] J. P. Mynard, P. Nithiarasu. A $1 D$ arterial blood flow model incorporating ventricular pressure, aortic valve and regional coronary flow using the locally conservative Galerkin (LCG) method. Comm. Num. Met. Eng., 24 (2008), 367-417.

[11] C. G. Caro, T. J. Pedley, R. C. Schroter, W. A. Seed. The Mechanics of the circulation. Oxford University Press, Oxford, New York, 1978.

[12] R. Sala, C. Rossel, P. Encinas, P. Lahiguera. Continuum of pulse wave velocity from young elite athletes to uncontrolled older patients with resistant hypertension. J. Hypertens, 28 (2010), 19.216.

[13] R. F. Schmidt, G. Thews. Human Physiology, vol.2, 2nd ed., MIR, Moscow, 1996 (In Russian).

[14] S. Simakov, T. Gamilov, Y.N. Soe. Computational study of blood flow in lower extremities under intense physical load. Russ. J. Numer. Anal. Math. Mod., 28 (2013), 485-504.

[15] S. Simakov, A. Kholodov. Computational study of oxygen concentration in human blood under low frequency disturbances. Mat. Mod. Comp. Sim., 1 (2009), 3-295.

[16] Y. Vassilevski, S. Simakov, S. Kapranov. A multi-model approach to intravenous filter optimization. Int. J. Numer. Meth. Biomed. Eng., 26 (2010), 915-925.

[17] Y. Vassilevski, S. Simakov, T. Dobroserdova. Numerical issues of modelling blood flow in networks of vessels with pathologies. Russ. J. Numer. Anal. Math. Mod., 26 (2011), 605-622.

[18] I. B. Wilkinson, J. R. Cockcroft, D. J. Webb. Pulse wave analysis and arterial stiffness. J. Cardiovasc. Pharmacol., 32 (1998), Suppl. 3, S33-7.

[19] http://www.vmtk.org

[20] http://www.amira.com, http://www.vsg3d.com/amira/

[21] http://www.plasticboy.co.uk/ 